\title{
MASS FUNCTIONS \& STELLAR POPULATIONS OF GLOBULAR CLUSTERS
}

\author{
P. GUHATHAKURTA \\ UCO/Lick Observatory, Univ. of California, Santa Cruz, CA 95064, USA \\ G. PIOTTO \\ Dipartimento di Astronomia, Vicolo dell'Osservatorio, 5, I-35122 Padova, Italy \\ AND \\ E. VESPERINI \\ Dept of Physics \& Astronomy, Univ. of Massachusetts, Amherst, MA 01003, USA
}

\section{Stellar content and density profiles of dense globular clusters through the eyes of the Hubble Space Telescope (P. Guhathakurta)}

\subsection{INTRODUCTION}

I present a summary of results from various $H S T$ photometric studies of the dense central regions of Galactic globular clusters that my collaborators and I have carried out over the last 6 years. The dataset includes short exposures of 47 Tuc, M15, M3 and M13 obtained with the aberrated Planetary Camera-I (PC-I) and F555W ("V") and F785LP ("I") filters, as well as post-refurbishment Wide Field Planetary Camera 2 (WFPC2) snapshots of the post core collapse clusters M15, M30, and NGC 6624 in F336W (" $U$ "), F439W (" $B$ "), and $V$. Recently, a very deep, doubly oversampled PC-I $U$ image of the core of 47 Tuc, and accompanying $B$ and $V$ images, have also been analyzed. In addition, we have carried out extensive checks of incompleteness and photometric error with the help of multiband image simulations that mimic the relevant characteristics of the HST PC-I and WFPC2 images: empirical point spread function, crowding effects based on a realistic density profile and stellar luminosity function (LF), noise, undersampling, A/D saturation, etc..

Our studies focus on: (1) The stellar density distribution near cluster centers as a probe of the dynamical state of the system; and (2) The mix of evolved stellar populations and the interplay between stellar evolution and encounters/interactions in dense environments. The data are deep enough to obtain complete samples of post main sequence even in the most crowded regions of clusters $\left(V_{\text {turnoff }} \sim 19\right.$, for a cluster at $D=10 \mathrm{kpc}$ ), but do not probe far enough down the main sequence LF to sample stars whose masses are substantially lower than that of red giants $\left(m_{\text {turnoff }} \approx 0.8 m_{\odot}\right)$. Thus, the issue of mass segregation (cf. King et al. 1995; Sosin \& King 1997; Piotto, next Sec.) is not addressed by our studies.

\subsection{RESULTS}

The salient results from our work are summarized below, with special emphasis on some of the questions that remain unanswered. Full details are given in the references cited below. Meylan \& Heggie (1996) give an extensive review of the subject.

1. Density Cusps in Post Core Collapse Clusters- Post core collapse clusters are characterized by surface brightness profiles that appear to rise all the way in to the limit of ground based resolution. Early studies based on pre-repair HST images appeared to resolve the center of M15 into a $r_{\text {core }}=2.2^{\prime \prime}$ region of constant density (Lauer et al. 1991) but the reliability of this result was challenged by Yanny et al. (1994a). A clearer picture emerged with the refurbished HST. Guhathakurta et al. (1996b) analyzed WFPC2 star counts in M15 in three ways and found a power law density profile and ruled out a $2^{\prime \prime}$ core, a result confirmed by Sosin \& King (1997) using FOC+COSTAR. These and other studies (cf. Yanny et al. 1994b; Guhathakurta 
et al. 1996a) indicate that the projected density profile of evolved stars in post core collapse clusters is well approximated by a power law of index -0.8 , consistent with core collapse and black hole model predictions. Another interesting line of research is the study of rotational flattening in the cores of dense clusters. For example, slight rotation $(v \sim 2 \mathrm{~km} / \mathrm{s})$ is observed in the central $15^{\prime \prime}$ of M15, and the stellar isodensity contours show a corresponding amount of ellipticity, $\epsilon \approx\left(v / \sigma_{v}\right)^{2} \approx 0.05$, aligned with the rotation axis (Guhathakurta et al. 1996b).

2. The Controversy Surrounding the Core Radius of 47 Tuc- The core radius of 47 Tuc has been the subject of debate during the last 5 years. Guhathakurta et al. (1992) measured $r_{\text {core }}=23^{\prime \prime}$ from PC-I $V$-band star counts, in agreement with previous ground based analyses of the light profile. Using pre-repair ultraviolet FOC and PC-I images, respectively, Calzetti et al. (1993) suggested that $47 \mathrm{Tuc}$ is on the verge of core collapse $\left(r_{\text {core }}<15^{\prime \prime}\right)$, while De Marchi et al. (1996) claimed the cluster is in post collapse reexpansion $\left(r_{\text {core }}=12^{\prime \prime}\right)$. The authors cite incompleteness and/or cluster centroid measurement error in earlier studies as the cause of the discrepancy. However, Guhathakurta et al. (1993) have shown that the effect of centroid error and incompleteness in the PC-I $V$-band star counts is negligible. Moreover, careful reanalysis of the same deep PC-I $U$ data used by De Marchi et al., based on PSF fitting instead of aperture photometry, reveals a normal, relaxed core, $r_{\text {core }} \gtrsim 20^{\prime \prime}$ (Guhathakurta et al. 1998) that shows no sign of collapse. The small apparent core radii found by Calzetti et al. and De Marchi et al. are a result of increased photometric error (neighbor contamination of aperture mags) near the crowded cluster center which causes many sub-turnoff stars to scatter into their samples.

3. Blue Straggler Stars- A lot of data has been gathered on blue straggler stars (BSSs) in recent times. The high angular resolution of $H S T$, in particular, has facilitated the study of dense cluster cores where BSSs preferentially reside. The nature of stragglers, though, continues to be somewhat of an enigma. For example, the specific frequency of BSSs relative to bright giants is in the narrow range of $5 \%-20 \%$ for clusters spanning several orders of magnitude in stellar density (and hence in collision probability). BSSs in cluster cores are centrally concentrated relative to giants, even in clusters where their global distribution appears to be bimodal (Ferraro et al. 1993; Guhathakurta et al. 1994). There is little correlation however between cluster core density and the degree of central concentration of BSSs: e.g., their relative concentration is very strong in M30 and 47 Tuc, but not in M3 or M15. Other lines of investigation include studies of the BSS LF as a discriminant of their formation mechanism (Bailyn \& Pinsonneault 1995; Guhathakurta et al. 1998; Webster et al. 1998) and variability (cf. Edmonds et al. 1996).

4. Deficiency of the Brightest Red Giants Near Dense Cluster Centers-- Color gradients near the centers of post core collapse globular clusters have long been thought to be due to the depletion of the brightest red giants in these very dense environments (Piotto et al. 1988). This is quantified by Webster et al. (1998) in their detailed investigation of M30's color gradient. The deficiency is usually strongest in the inner $10^{\prime \prime}-20^{\prime \prime}$, with the bright red giant fraction at the center being $30 \%-50 \%$ of the value observed in the outer parts; the strength and significance (based on Poisson statistics) of the effect depends on the exact definition of the bright red giant sample. It is noteworthy that horizontal branch stars, the downstream evolutionary products of red giants, are not as strongly depleted in the central regions of the same clusters (Guhathakurta et al. 1996c); this is suggestive of short-circuiting of (rather than termination at) the bright red giant phase in dense regions.

5. Shape of the Luminosity Function of Evolved Stars- The shape of the LF of evolved stars can serve as a useful test of standard stellar evolutionary models. Groundbased studies noted a mild excess of giants relative to turnoff/main sequence stars in the relatively sparse regions of M30; the effect is also seen near the cluster center (Webster et al. 1998). The excess can be explained by invoking the effects of internal rotation in stars. However, a cluster where internal stellar rotation is expected to be even more prominent is 47 Tuc, and its LF is a good match to the standard model (Guhathakurta et al. 1998). A recent study of M13's LF reveals a different kind of discrepancy: there is an excess of only the brightest red giants $\left(V<V_{\mathrm{HB}}\right)$ relative to the standard model (Cohen et al. 1997). Could this be related to the deep mixing observed in this cluster (via anomalous elemental abundances of the brightest giants)?

I would like to acknowledge the contributions of my collaborators on various globular cluster projects: J. Bahcall, R. Cohen, P. Edmonds, R. Gilliland, D. Schneider, Z. Webster, and B. Yanny. 


\subsection{REFERENCES}

Bailyn, C.D., \& Pinsonneault, M.H., 1995, ApJ, 439, 705

Calzetti, D., De Marchi, G., Paresce, F., \& Shara, M., 1993, ApJ, 402, L1

Cohen, R.L., Guhathakurta, P., Yanny, B., Schneider, D.P., \& Bahcall, J.N., 1997, AJ, 113, 669

De Marchi, G., Paresce, F., Stratta, M.G., Gilliland, R.L., \& Bohlin, R.C., 1996, ApJ, 468, L51

Edmonds, P.D., Gilliland, R.L., Guhathakurta, P., Petro, L.D., Saha, A., \& Shara, M.M., 1996, ApJ, 468, 241

Ferraro, F.R., Fusi Pecci, F., Cacciari, C., Corsi, C., Buonanno, R., Fahlman, G.G., \& Richer, H.B., 1993, AJ, 106, 2324

Guhathakurta, P., Gilliland, R.L., \& Edmonds, P.D., 1998, AJ, in prep

Guhathakurta, P., Yanny, B., Bahcall, J.N., \& Schneider, D.P., 1994, AJ, 108, 1786

Guhathakurta, P., Yanny, B., Bahcall, J.N., \& Schneider, D.P., 1996a, in Dynamical Evolution of Star Clusters, eds. P. Hut \& J. Makino (Kluwer: Dordrecht), 333

Guhathakurta, P., Yanny, B., Schneider, D.P., \& Bahcall, J.N., 1992, AJ, 104, 1790

Gubathakurta, P., Yanny, B., Schneider, D.P., \& Bahcail, J.N., 1993, in Blue Stragglers, ed. R.A. Saffer (ASP Conference Series, Vol. 53), 60

Guhathakurta, P., Yanny, B., Schneider, D.P., \& Bahcall, J.N., 1996b, AJ, 111, 267

Guhathakurta, P., Yanny, B., Schneider, D.P., \& Bahcall, J.N., 1996c, in Dynamical Evolution of Star Clusters, eds. P. Hut \& J. Makino (Kluwer: Dordrecht), 19

King, Y.R., Sosin, C., \& Cool, A.M., 1995, ApJ, 452, L33

Lauer, T.R., et al., 1991, ApJ, 369, L45

Meylan, G., \& Heggie, D.C., 1997, A\&A Rev., 8, 1

Piotto, G., King, I.R, \& Djorgovski, S., 1988, AJ, 96, 1918

Sosin, C., \& King I.R., 1997, AJ, 113, 1328

Webster, Z.T., Guhathakurta, P., Yanny, B., Schneider, D.P., \& Bahcall, J.N., 1998, AJ, in prep

Yanny, B., Guhathakurta, P., Bahcall, J.N., \& Schneider, D.P., 1994a, AJ, 107, 1745

Yanny, B., Guhathakurta, P., Schneider, D.P., \& Bahcall, J.N., 1994b, ApJ, 435, L59

\section{A Comparison of Deep HST Luminosity Functions (G. Piotto)}

In the last couple of years, thanks to HST, we have been able to investigate the properties of the globular cluster $(\mathrm{GC})$ luminosity functions (LF) and of the resulting mass functions (MF) down to $\leq 0.10 m_{\odot}$, i.e. nearly the hydrogen-burning limit. The MFs of GC stars give insight into the formation and dynamical evolution of globulars, and on the Galactic halo star population.

Here we present the LFs from HST/WFPC2 data for a sample of 6 metal poor and 2 intermediate metallicity clusters. The LFs and MFs of M15 (NGC 7078), M30 (NGC 7099), M92 (NGC 6341), and NGC 6397 have already been discussed by Piotto et al. (1997); the MF of NGC 6752 is from Ferraro et al. (1997); the LFs and MFs of M10 (NGC 6254), M22 (NGC 6656), and M55 (NGC 6809) will appear in Piotto \& Zoccali (1998, in prep).

All the LFs have been obtained from color-magnitude diagrams (CMD), which allow the direct discrimination of cluster stars from field objects. Fig. 1 shows an example of the CMDs we have been working on. Note that the deep main sequence from the HST images have been extended to brighter magnitudes with ground-based data. For the first time, we can show a CMD which extends from just above the hydrogen-burning limit to the tip of the giant branch and down to the white dwarf sequence, i.e. covering all the presently observable evolutionary phases of a GC star. This is powerful tool for a fine tuning of the stellar evolution models. Ground-based data allows also to extend the LFs to the turn-off. The LFs presented here cover the entire mass range of the GC main sequence stars, $\left(0.1 \leq m / m_{\odot} \leq 0.8\right)$ : this is very important when we want to compare the LFs in different clusters (cf. Piotto et al. 1997, for a discussion).

Six of the clusters mentioned above share two features which make for a particularly useful comparison. First, with the exception of M10 and NGC 6752, all the clusters have comparable metallicity $([\mathrm{Fe} / \mathrm{H}]=-2.0 \pm 0.2)$, so that similarities or differences in their LFs will directly reflect similarities or differences in their MFs. Second, for all of them, the observed (local) LF do not differ appreciably from the global one. This has been explicitly tested. For the three King-model profle clusters (M22, M55, M92) we ran multimass King-Michie models (Piotto \& Zoccali 1998): in all the cases, the local MF resulted very similar to the global one. This is a consequence of the fact that the HST fields for these objects are located close to their half-mass radius, i.e. close to the cluster region where the local and global MFs almost concide. For the three post-core-collapse clusters (M15, M30, and NGC 6397) see the discussion in Piotto et al. (1997).

The V LFs for the six metal poor clusters are compared in the left panel of Fig 2. Vertical shifts were made to bring the LFs into alignment according to a least-squares algorithm in the magnitude interval $4.0<V<7.0$. The broad features of these LFs are similar: they all rise to a 


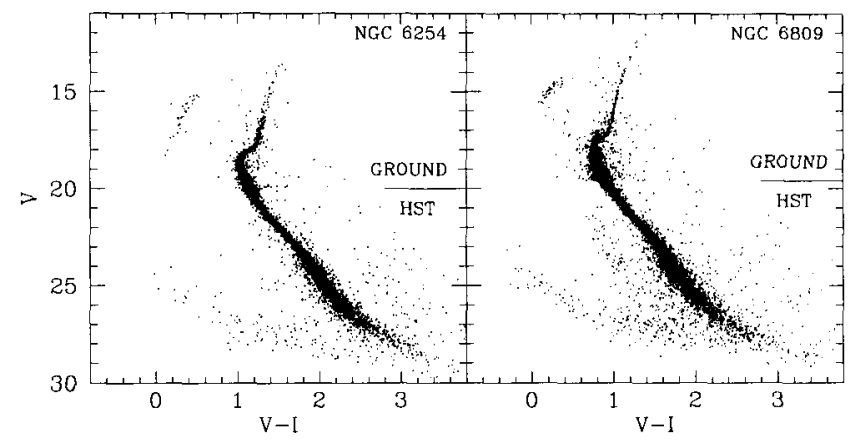

Figure 1. The CMDs of M10 and M55. The diagram for magnitudes fainter than $\mathrm{V}=20$ is from WFPC2/HST data; brighter stars are from ground-based images. For the first time we have a GC CMD covering all the observable evolutionary sequences of the GC stars, from just above the hydrogen-burning limit to the white dwarf sequence.
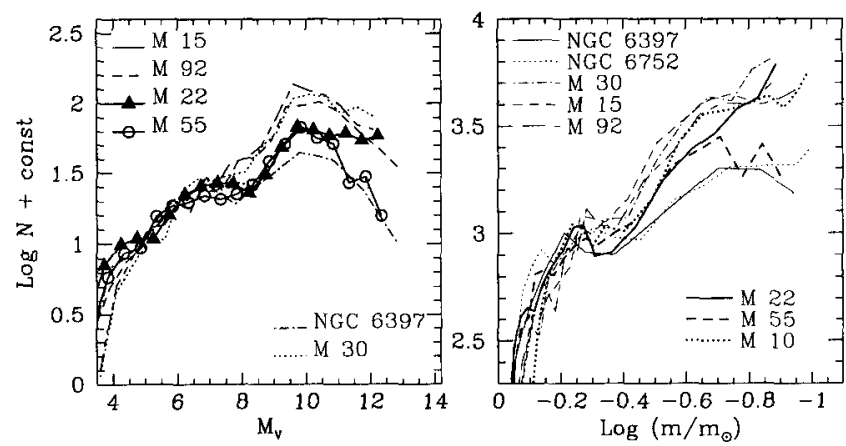

Figure 2. Left panel: The LFs for the six metal poor GCs. The LFs span a large range of slopes, with M30-M15 and NGC 6397 at the two extremes; Right panel: the MFs for the six clusters in the left panel plus M10 and NGC 6752.

peak near to $V \sim 9.5-10.5$, then bend over and drop significantly to the limit of the observations (with the only exception of the LF of M22 which seems to level off at the faint magnitudes). As noticed by Piotto et al. (1997), the LF of NGC 6397 is the most deficent in faint stars. The new LFs of M22 and M55 seem to have an intermediate behaviour, filling the gap between NGC 6397 and the steepest LFs of M15, M30, and M92. The LFs in Fig 2 might simply reflect the fact that these clusters are born with different MFs. Piotto et al. (1997) give a different interpretation. In view of the similarities of the LFs of M15, M30, and M92, and of the fact that NGC 6397 has the shortest central relaxation time, and an orbit that is among the most vulnerable to tidal shocks, they suggest that the observed differences are due to tidal shocks and ejection through internal relaxation. We still think that this is the most plausible interpretation for Fig 2 . The orbit of M22 is an intermediate case between the orbit of M15 and NGC 6397 (Dauphole et al. 1996); moreover, though no orbit is presently available for M55, we know that this cluster is presently losing stars (Zaggia et al. 1997). As shown by E. Vesperini in the next Section, evaporation and tidal shocks can significantly change the GC global MFs in a Hubble time.

The right panel of Fig 2 shows the MFs for the six metal poor clusters plus M10 and NGC 6752 . Again, the MFs show different slopes, with M30 (the steepest one) and NGC 6397 (the flattest one) at the two extremes. It is also notewhorty that the striking drop of all the LFs at the faint end is absent in the MF. In all the cases, the steepness of the theoretical mass-luminosity relation for the faintest stars has largerly compensated for it. In no case there is a clear evidence of a drop off of the MF at the lowest masses, with the possible exception of NGC 6397.

\subsection{REFERENCES}

Dauphole, B., Geffert, M., Colin, J., Ducourant, C., Odenkirchen, M., \& Tucholke, H.J., 1996, 
A\&A, 313, 119

Ferraro, F.R., Carretta, E., Bragaglia, A., Renzini, A., \& Ortolani, S., 1997, MNRAS, 286, 1012

Piotto, G., Cool, A.M., \& King, I.R., 1997, AJ, 113, 134

Zaggia, S., Piotto, G., \& Capaccioli, M., 1997, A\&A, in press

\section{On the effects of dynamical evolution on the initial mass function of globular clus- ters (E. Vesperini)}

\subsection{INTRODUCTION}

Many observational and theoretical investigations have beeen carried out to study different issues regarding the mass function of globular clusters but many points such as the shape of the initial mass function (IMF), the dependence of the present-day mass function (PDMF) on cluster parameters, the actual relevance of dynamical evolution in determining the shape of the PDMF and the relation between the IMF and the PDMF are still matters of debate.

While it has been suggested in some works (see e.g. De Marchi \& Paresce 1995 and references therein) that differences in the metallicity would be the main factor responsible for the observed differences in the mass functions of clusters, with dynamical evolution playing no relevant role, as discussed in Piotto, Cool \& King (1997) some recent HST results seem to point to an important role of evolutionary processes in altering the IMF of some Galactic globular clusters.

The main goal of our theoretical investigation is that of assessing the importance of various evolutionary processes in altering the mass function of a globular cluster and that of understanding to what extent these processes can be responsible for the differences observed between the mass functions of Galactic globular clusters. Since in our simulations we have taken into account the main effects of stellar evolution, we have also addressed some issues concerning the stellar content of globular clusters, focussing our attention in particular on the fraction of white dwarfs expected to be present in a cluster during the different stages of its dynamical evolution.

We have carried out a large set of $N$-body simulations using a version of the code NBODY4 (see e.g. Aarseth 1985) which includes the effects of the presence of the tidal field of the Galaxy, stellar evolution, disk shocking, two-body relaxation; the initial conditions adopted span a range of different values of the mass and concentration of the cluster, the slope of the IMF and the distance from the Galactic center. Most of the simulations have an initial number of particles $N=4096$ but some runs with $N=8192$ and $N=16384$ have been carried out to study the dependence of the results on the number of particles in the simulation. Disk shocking has been included according to the model described in Chernoff, Kochanek \& Shapiro (1986) and as for stellar evolution we have adopted the same model used in Chernoff \& Weinberg (1990).

We have assumed the clusters to be on circular orbits and to move in a Keplerian potential determined by a point mass $M_{g}$ equal to the total mass of the Galaxy inside the adopted galactocentric distance $R_{g}$; multi-mass King models with different concentrations have been adopted as initial conditions, masses of stars have been assigned according to a power-law mass function $d N(m)=m^{-\alpha} d m$ between $0.1 m_{\odot}$ and $15 m_{\odot}$ and initially there is no equipartition of energies of stars with different masses. An initial value of $\alpha$ equal to 2.5 has been adopted in most runs; some runs with $\alpha=3.5$ have been carried out in order to investigate the dependence of the final results on the initial slope of the mass function.

\subsection{RESULTS}

The main results of our work are (see Vesperini \& Heggie 1997 for a complete and more detailed discussion of our investigation and of the results obtained):

1. As a result of mass loss through the tidal boundary, both due to two-body relaxation and to disk shocking, the global mass function becomes flatter. For given initial parameters, mass loss is stronger for clusters closer to the Galactic center, and, consequently, a trend between the slope of the MF and the galactocentric distance (similar to that suggested by the analysis of observational data by Capaccioli et al. (1993) and Djorgovski et al. (1993)) forms as evolution goes on. This trend is stronger for low-mass clusters, as these have shorter relaxation times and thus evolve more quickly than massive clusters. Both mass loss by two-body relaxation and disk shocking are important in causing the MF to flatten. 
The difference between the initial and the final (at $t=15 \mathrm{Gyr}$ ) slope of the MF has been shown to depend approximately only on the fraction of the initial mass lost and this dependence is the same no matter whether disk shocking is included or not.

The evolution of the slope of the MF is slower for steeper IMFs.

By means of the results of $N$-body simulations it has been possible to derive an analytical expression for the slope of the mass function at any time $t$ and for any initial value of the mass and of the galactocentric distance both with and without the effects of disk shocking.

2. The MF near the half-mass radius is the one which, during the entire evolution, is least affected by mass segregation and quite well resembles the present-day global mass function. The extent to which the MF observed near the half-mass radius can provide us with useful information on the IMF thus depends on the difference between the global IMF and the global PDMF. Possibly, when the the PDMF is different from the IMF, observations of the MF at radii larger than the half-mass radius can supply indications on the IMF, but it is important to note that, in cases of strong mass loss and evolution of the IMF, we have shown that, even the MF in the outer regions of the clusters eventually becomes flatter than the IMF, whose memory is thus completely erased from the PDMF. The MF in the inner regions is always significantly flatter than the PDMF as a result of mass segregation.

3. The ratio of the total mass of white dwarfs retained in the cluster to the total mass of the cluster, $M_{w d} / M(t)$, increases during the evolution: as the fraction of the initial mass left in the cluster decreases, the fraction of this in white dwarfs increases. The total mass of white dwarfs is determined by the intexplay between the rate of production determined by the time scales of stellar evolution and the rate of escape through the tidal boundary of the white dwarfs, or possibly of their progenitors, determined by the relaxation time and disk shocking time scale. We have made a comparison between our estimate of the fraction of white dwarfs and the one which would be obtained by extrapolating the present day main sequence mass function (a procedure often used in literature) and we have shown that the former is, in most cascs, smaller than the latter, with the ratio of the two estimates ranging, in most relevant cases, from about 0.65 to 1 depending on the initial conditions.

We have obtained an analytical expression for the fraction of white dwarfs present in a cluster as a function of the initial conditions and of time; this depends on the production rate, that can be easily derived analytically once the IMF has been given, and the escape rate which is derived instead from a fit to the $N$-body data.

\subsection{REFERENCES}

Aarseth, S.J., 1985, in Multiple Time Scales, eds. J.U. Brackbill \& B.I. Cohen (Academic Press, New York), 377

Capaccioli, M., Piotto, G., \& Stiaveli, M., 1993, MNRAS, 261, 819

Chernoff, D.F., Kochanek, C.S., \& Shapiro, S.L., 1986, ApJ, 309, 183

Chernoff, D.F., \& Weinberg, M.D., 1990, ApJ, 351, 121

De Marchi, G., \& Paresce, F., 1995, A\&A, 304, 211

Djorgovski, S., Piotto, G., \& Capaccioli, M., 1993, AJ, 105, 2148

Piotto, G., Cool, A.M., \& King, I.R., 1997, AJ, 113, 1345

Vesperini, E., \& Heggie, D.C., 1997, MNRAS, 289, 898 